\title{
Heterogeneity of Spacer Lengths in Circles of Amplified Ribosomal DNA of Two Insect Species, Dytiscus marginalis and Acheta domesticus
}

\author{
Michael F. Trendelenburg, Ulrich Scheer, Hanswalter Zentgraf \\ and Werner W. Franke \\ Division of Membrane Biology and Biochemistry \\ Institute of Experimental Pathology and \\ Institute of Virology, German Cancer Research Center \\ D-69 Heidelberg, Federal Republic of Germany
}

(Received 1 June 1976, and in revised form 28 July 1976)

The amplified, extrachromosomal rDNA $†$ in the oocytes of two taxonomically unrelated species, the water beetle Dytiscus marginalis and the house cricket Acheta domesticus, has been studied with two different electron microscopic techniques. (1) The patterns of transcribed and fibril-covered genes for pre-rRNA (matrix units) and the interspersed, fibril-free apparent spacer intercepts have been analysed in spread and positively stained preparations of nucleolar chromatin from manually isolated nuclei. (2) The contour lengths of circular molecules or isolated rDNA from such nuclei as revealed by surface spreading with cytochrome $c$ have been measured. (3) In addition, the sizes of the pre-rRNA molecules, as determined by gel electrophoresis, have been compared with the sizes of the matrix units involved in pre-rRNA formation. In both species a significant amount of the rDNA has been recovered in closed circles containing from one to six pre-rRNA genes. In both species the information content of the transcribed regions exceeds that of the pre-rRNA molecules $\left(2.8 \times 10^{6}\right.$ molecular weight) considerably (35\% in Dytiscus, about $100 \%$ in Acheta), indicative of an instability of the true primary transcription products. While the matrix units are relatively homogeneous in length (especially in Dytiscus), the apparent spacer intercepts as well as the resulting repeating units show a pronounced heterogeneity with indications of some preferential subclasses. This heterogeneity can be classified into inter-axial (including intercircular) and intra-axial (including intracircular) heterogeneity of gene and spacer regions. Different circles can consist of different, although for a given circle identical, repeating units. In addition, circles containing repeating units of different lengths are also found. The results are discussed in relation to analyses of rDNA gene-spacer patterns in other organisms. The data show that the character and the pattern of heterogeneity of functional intercepts in rDNA are not uniform in the rDNA of a specific organism but may differ from one group of rDNA genes, or from one amplified rDNA molecule, to another. They further strongly suggest that circles of rDNA can be derived from different regions of one nucleolar organizer.

$\dagger$ Abbreviations used: rDNA, DNA that is enriched in, and may exclusively consist of, the genes for the common precursors to $18 \mathrm{~S}$ and $28 \mathrm{~S}$ rRNAs and the interspersed apparent spacer regions; $\mathrm{S}$ values of ribosomal RNAs (rRNAs) are nominal; pre-rRNA, the largest size class of stable molecules that contain $28 \mathrm{~S}$ and $18 \mathrm{~S}$ rRNA sequences; "apparent spacers", intercepts in rDNA chromatin strands which lie between the matrix units, i.e. the fibril-covered regions of transcriptional units of the pre-rRNA; spacers, regions in repeating units of rDNA that do not contain sequences complementary to pre-rRNA or to RNA which is transcribed from the same promoter as the pre-rRNA; repeating unit, the unit consisting of a transcriptional unit for prerRNA and the adjacent (subsequent or preceding) spacer. 


\section{Introduction}

The genes coding for the precursors to the two large ribosomal RNAs, $28 \mathrm{~S}$ and $18 \mathrm{~S}$ rRNA $†$, are especially suitable for analyses of gene arrangement and gene activity because they occur in relatively high numbers of copies and are clustered in the nucleolar organizer regions of the chromosomes or in extrachromosomal units (for references see below). Moreover, the rDNA of many organisms can be prepared in relatively pure form, especially in those cells in which it differs in buoyant density from the bulk nuclear DNA and/or in which it is enriched in amplified copies, such as in the oocytes of various amphibia and insects and in some lower eukaryotes (see e.g. Gall et al., 1969; Dawid et al., 1970; Birnstiel et al., 1971; Cave, 1972,1973; Macgregor, 1973; Ullman et al., 1973; Engberg et al., 1974; Gall, 1974; Bohnert et al., 1975). In addition, it is possible to study these genes by electron microscopy as both isolated rDNA (for references see above) and transcribed nucleolar chromatin (Miller \& Beatty, 1969a,b), as well as at different stages of transcriptional activity (Scheer et al., 1975,1976).

For some years there were the prevailing hypotheses that these genes (1) occur in the form of a repeating unit of similar length in all eukaryotes (cf. Perry et al., 1970), and (2) are arranged in highly homogeneous patterns, i.e. with constant ratios of the lengths of gene regions and apparent spacer intercepts that are characteristic of the specific groups of organisms or the specific species (Wensink \& Brown, 1971; Brown et al., 1972; Miller \& Bakken, 1972; Brown \& Sugimoto, 1974; Forsheit et al., 1974). Recent studies, however, using various electron microscopic and biochemical techniques, have demonstrated (i) large differences in the lengths of rDNA repeating units both among different organisms and among different strands of nucleolar chromatin of the same cell (Scheer et al., 1973; Trendelenburg et al., 1973,1974,1975; Spring et al., 1974,1976; Trendelenburg, 1974; Berger \& Schweiger, 1975a,b) and (ii) heterogeneities in the "gene-spacer" pattern within one species and even within the same nucleus (for references see Scheer et al., 1973; Morrow et al., 1974; Spring et al., 1974,1976; Trendelenburg et al., 1974; Wellauer \& Dawid, 1974; Wellauer et al., 1974; Wellauer \& Reeder, 1975). Such heterogeneity was observed with both chromosomal and extrachromosomal rDNA copies (Wellauer \& Reeder, 1975) but has hitherto only been described in linear molecules.

A special form of rDNA is provided by the circles of amplified rDNA as they have been observed in oocytes and somatic cells of the clawed toad Xenopus laevis (Miller \& Beatty, 1969b; Hourcade et al., 1973; Bakken, 1975; Rochaix \& Bird, 1975; Buongiorno-Nardelli et al., 1976), in the oocytes of the newts Triturus alpestris (Scheer et al., 1976) and Pleurodeles walti (Angelier \& Lacroix, 1975), and in the oocytes of two insect groups, the water beetles genera Dytiscus and Colymbetes (Gall \& Rochaix, 1974; Trendelenburg, 1974) and the house cricket Acheta domesticus (Trendelenburg et al., 1976; J. G. Gall, personal communication). Circular rDNA has also been reported in the slime mold Physarum polycephalum (Bohnert et al., 1975), and in the macronuclei of the ciliate Tetrahymena pyriformis (Gall, 1974). Moreover, it has been suggested that such circles occur, at least transiently, in the amplification process (Hourcade et al., 1973; Rochaix et al., 1974; Bakken, 1975; Rochaix \& Bird, 1975; Buongiorno-Nardelli et al., 1976). In all studies of such circular forms of rDNA the homogeneity of the gene-spacer pattern and of the length of the repeating unit has been emphasized (see e.g. Gall \& Rochaix, 1974; Rochaix

+ See footnote p. 453. 
et al., 1974; Bakken, 1975; Rochaix \& Bird, 1975; Buongiorno-Nardelli et al., 1976). In the present study we demonstrate heterogeneities of the lengths of the apparent spacer intercepts and the repeating units in the circles of amplified rDNA from two insect species, one representative of those with a meroistic ovary, Dytiscus marginalis, and one with a panoistic ovary, Acheta domesticus. On the basis of electron microscopic determinations in both transcribed nucleolar chromatin and in isolated rDNA, we also show that rDNA rings occur with both homogeneous patterns and with intracircular heterogeneity.

\section{Materials and Methods}

(a) Biological material

D. marginalis females were either collected in the vicinity of Heidelberg or were kindly provided by Dr H. Schildknecht (University of Heidelberg). They were kept in large tanks in running tap water at about $16^{\circ} \mathrm{C}$. The house crickets, $A$. domesticus, were reared in the laboratory and were provided by Dr H. J. Bode (University of Heidelberg).

\section{(b) Spread preparations of nuclear contents}

Nuclei from early vitellogenic oocyte stages were manually isolated as described previously (Trendelenburg, 1974; Trendelenburg et al., 1973,1976). The nuclear content was allowed to disperse under very low salt conditions $(0 \cdot 1 \mathrm{mu}$-borate buffer, $\mathrm{pH} 9 \cdot 0)$ at about $12^{\circ} \mathrm{C}$ for 10 to $30 \mathrm{~min}$ (ef. Miller \& Bakken, 1972; Spring et al., 1974). Some preparations were rotary shadowed with $\mathrm{Au} / \mathrm{Pd}$ at an angle of $7^{\circ}$ after positive staining with phosphotungstic acid (Scheer et al., 1976).

\section{(c) Isolation and spreading of $r D N A$ for electron microscopy}

About 100 oocyte nuclei were collected in ice-cold $70 \%$ ethanol. Usually the manually isolated nuclei were still surrounded by a thin layer of tightly adhering, juxtanuclear ooplasm. The sedimented material was lysed in $4 \mathrm{ml}$ of a solution containing $50 \mathrm{~mm}$ Tris-HCl (pH 8.4), 20 mm-EDTA, 1\% Sarkosyl NL-97 (Ciba-Geigy, Basel, Switzerland) and $0.5 \mathrm{mg}$ pre-digested Proteinase $\mathrm{K} / \mathrm{ml}$ (Merck, Darmstadt, Federal Republic of Germany) at $37^{\circ} \mathrm{C}$ (cf. Gall \& Rochaix, 1974). After $2 \mathrm{~h}, 0.5 \mathrm{mg}$ pre-digested Pronase $/ \mathrm{ml}$ (Calbiochem, San Diego, Calif., U.S.A.) was added, and the solution was incubated with gentle shaking for $3 \mathrm{~h}$ at $37^{\circ} \mathrm{C}$. CsCl was then added directly to a final refractory index of $1 \cdot 3998$ and the DNA was centrifuged to equilibrium as described by Gall \& Rochaix (1974). The gradient was fractionated into 5-drops portions. Either total DNA (buoyant density range from 1.687 to $1.735 \mathrm{~g} \mathrm{~cm}^{-3}$ ) was pooled or the DNA from the rDNA-containing peak fractions (Gall \& Rochaix, 1974) was collected, precipitated with 3 vol. $70 \%$ ethanol, and dissolved in $50 \mu \mathrm{l} 10 \mathrm{mM}$-Tris buffer (pH 7.2), 1 mM-EDTA. The DNA was spread for electron microscopy by the cytochrome $c$ droplet diffusion method (Lang $\&$ Mitani, 1970). One droplet contained $200 \mu \mathrm{l} 0 \cdot 2 \mathrm{M}$-ammonium acetate, $2 \mu 10 \cdot 1 \%$ cytochrome $c$, and 2 to $5 \mu \mathrm{l}$ of the DNA solution. After a diffusion time of about $30 \mathrm{~min}$, the molecules were picked up on parlodion-coated grids, stained with uranyl acetate (Davis et al., 1971), dried from $90 \%$ ethanol, and were then rotary shadowed with $\mathrm{Pt} / \mathrm{Pd}(80: 20)$ at an angle of $8^{\circ}$. In some experiments the DNA was spread from a solution containing $50 \%$ formamide (or $80 \%$ formamide and $4 \mathrm{~m}$-urea) onto a hypophase of bidistilled water (ef. Davis et al., 1971). For comparison and estimation of molecular weights, defined circular DNA molecules (from bacteriophages $\phi \mathrm{X} 174$ and PM2 and simian virus 40 , the latter two in relaxed forms) were used in mixtures with the rDNA samples or in parallel preparations. Because of the great molecular size homogeneity, relaxed. PM2 DNA $\left(3.061 \pm 0 \cdot 19 \mu \mathrm{m}\right.$; corresponding to a molecular weight of $6.3 \times 10^{6}$, ef. Espejo et al., 1969) was taken for the estimations of the rDNA sizes presented in this study.

\section{(d) Electron microscopy and length measurements}

Mierographs were taken with a Zeiss EM-10 electron microscope at 40 or $60 \mathrm{kV}$. The magnification indicator was routinely controlled by comparison with a grating replica. 
The plates were projected onto a digitizer tablett, and the contour lengths of the DNA molecules or rDNA-containing chromatin intercepts were determined with the image analyzing system MOP-AM-O1 (Kontron Messgeräte, Eching bei München, Federal Republic of Germany). The length of a matrix unit was defined as the axial length within a matrix unit, i.e. the intercept between the smallest identifiable lateral fibril and the attachment site of the terminal fibril of the same fibril length gradient (cf. also Scheer et al., 1973; Franke et al., 1976a). Repeating units were determined as the intercepts consisting of a matrix unit plus (1) the preceding and/or (2) the subsequent spacer interval. Only repeating units with readily traceable axes were measured, and only completely traceable nucleolar circles were considered for the evaluation. Calculations and distribution analyses were made with the use of a computer program kindly developed by R. Zentgraf, Dipl. Math. (Institute of Documentation and Statistics, this centre).

\section{(e) Gel electrophoresis of nuclear RNA}

Individual Dytiscus females were anaesthetized with $\mathrm{CO}_{2}$, and $300 \mu \mathrm{Ci}\left[{ }^{3} \mathrm{H}\right]$ uridine (dissolved in a total of $50 \mu \mathrm{l}$ of $0.9 \% \mathrm{NaCl}$; spec. act. $45 \mathrm{Ci} / \mathrm{mmol}$; the Radiochemical Centre, Amersham, England) was injected into the body cavity. After $24 \mathrm{~h}$, the beetle was killed, and nuclei of early to middle vitellogenic oocyte stages were isolated and collected in ice-cold ethanol/acetic acid (3:1). The nuclei were washed in cold $70 \%$ ethanol containing $0 \cdot 1 \mathrm{M}-\mathrm{NaCl}$ and dissolved in $0.5 \mathrm{ml}$ of a solution containing $20 \mathrm{~mm}$-Tris $\cdot \mathrm{HCl}$ (pH 7.4), $0.5 \%$ sodium dodecyl sulphate, and $1 \mathrm{mg}$ predigested Pronase $/ \mathrm{ml}$ (Calbiochem) at room temperature for $30 \mathrm{~min}$. After addition of $20 \mu \mathrm{g}$ Escherichia coli rRNA and $10 \mu \mathrm{g}$ tobacco mosaic virus (TMV) RNA as molecular weight standards, the total nucleic acids were precipitated by adding 2 vol. absolute ethanol at $-20^{\circ} \mathrm{C}$. The pellet was dissolved in about $20 \mu \mathrm{l}$ diluted $(1: 1)$ electrophoresis buffer containing $10 \%$ sucrose and laid on top of $2.4 \%$ acrylamide gels in Plexiglas tubes. Conditions of electrophoresis were as described by Loening (1969); the electrophoresis buffer contained $36 \mathrm{~mm}$-Tris·HCl $(\mathrm{pH} 7 \cdot 6)$, $30 \mathrm{~mm}-\mathrm{NaH}_{2} \mathrm{PO}_{4}, 1$ mm-EDTA, $0 \cdot 2 \%$ sodium dodecyl sulphate. Gels were scanned at $260 \mathrm{~nm}$, sliced, and the radioactivity was determined (cf. Spring et al., 1976).

\section{Results}

(a) Morphology of transcribed rDNA and distribution of lengths of matrix units and apparent spacer intercepts

When nuclear contents from diplotene oocytes of $D$. marginalis were manually isolated and immediately and gently dispersed in low salt buffer and spread for electron microscopical observation (see Materials and Methods), we consistently found that the major part of the rDNA (more than $70 \%$ of the traceable nucleolar chromatin strands) was recovered in the form of small, extrachromosomal circular units (Figs 1 and 2 ; cf. Trendelenburg et al., 1976). Practically all of these rDNA strands appeared in a fully active form, i.e. they showed fully fibril-covered prerRNA gene regions (matrix units; Figs 1 and 2). The contour lengths of such circles were widely variable (Figs 1, 2 and 3). The largest clearly traceable circles contained up to five or six genes (Fig. 1), but rings comprising one or two genes were particularly frequent (Figs 1 and 2). In view of the good recovery of rDNA in ring-like units (see above), it is obvious that the frequency of such small rings can only partially be due to the lower susceptibility of small units to mechanical stresses and may well reflect the true predominance of small rDNA circles in vivo. In different preparations, an average of about $60 \%$ of the matrix units located on circles were in units containing one, two or three transcriptional units, i.e. pre-rRNA genes (Fig. 2).

Examination of several hundreds of such transeribed rDNA units revealed the occurrence of different lengths of the apparent spacer intercepts (Figs 1 and 2). A 


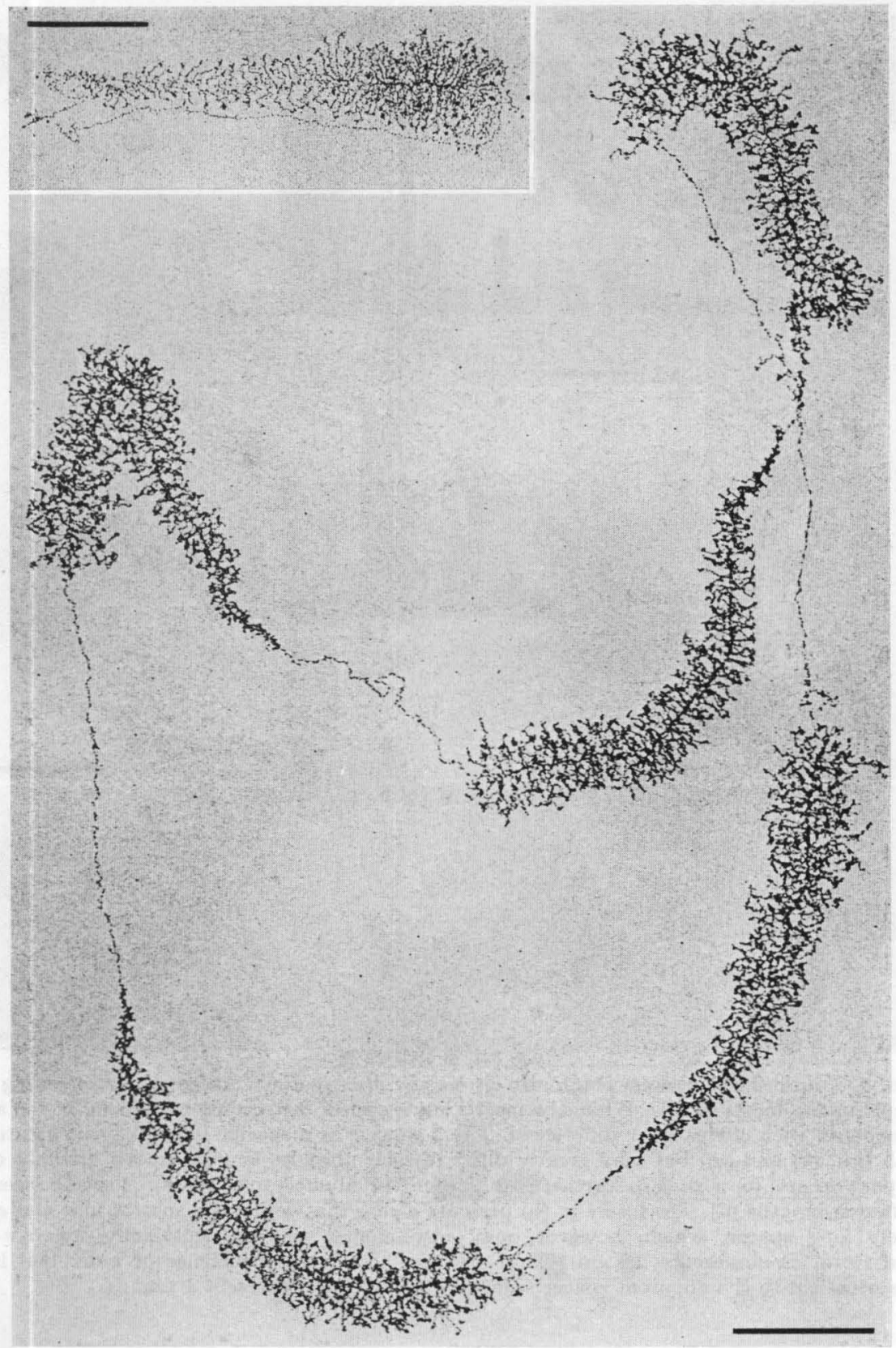

FIG. 1. Eleetron micrographs of a very large and a small (insert) example of the transeriptionally active nucleolar chromatin rings isolated from early vitellogenic Dytiscus oocytes as revealed in spread and positively stained preparations. The circles shown consist of 5 and 1 (insert) repeating units of rDNA and reveal the typical arrangement in transcribed and fibril-covered regions (matrix units) and interspersed apparent spacer intercepts. Note the nearly uniform length of the matrix units and the differences in the apparent spacer intercepts within the large circle. This large circle has a contour length of about $34 \mu \mathrm{m}$. Bars represent $1 \mu \mathrm{m}$. 


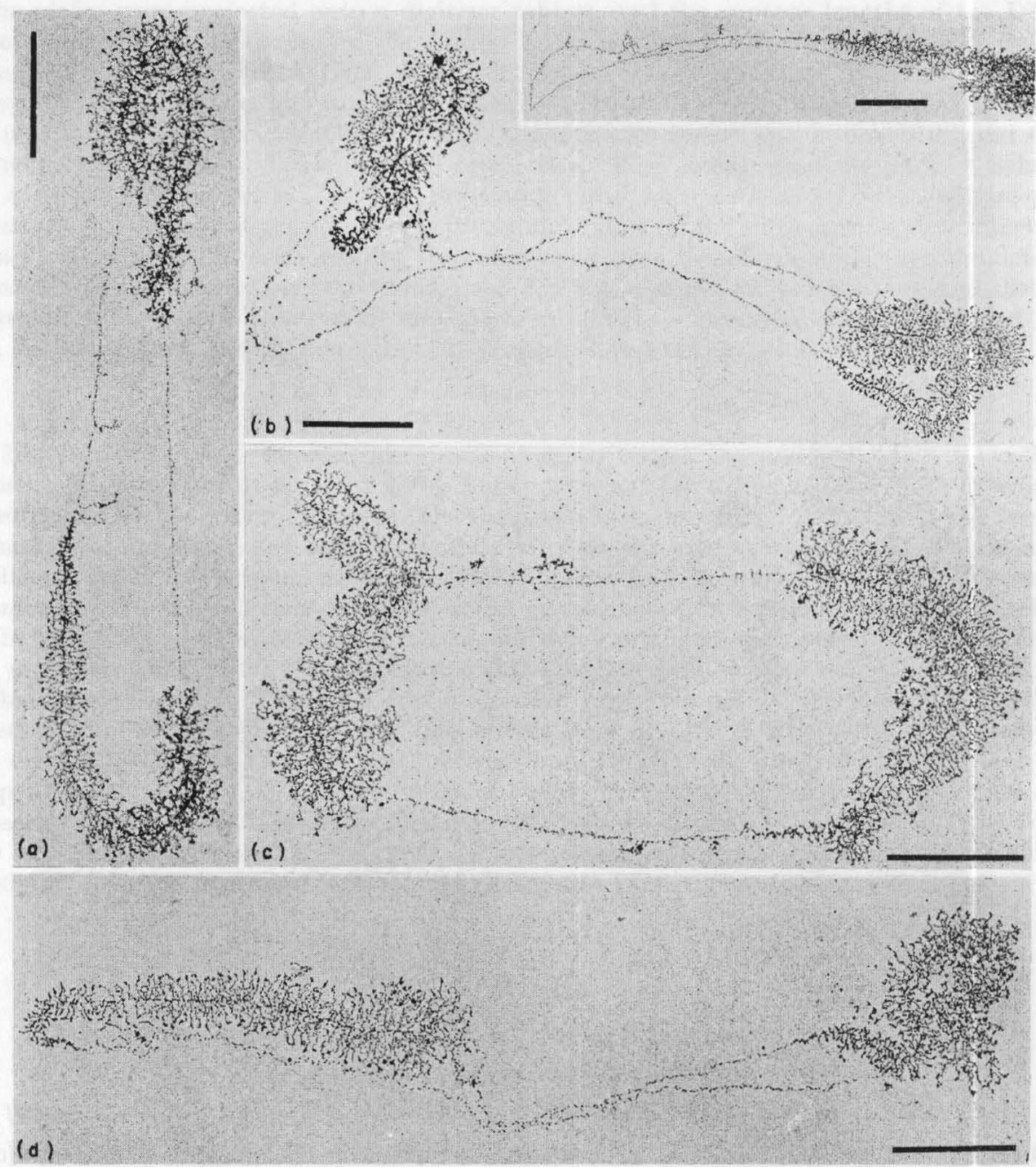

FIG. 2. Transcribed nucleolar chromatin circles (D. Marginalis) containing two repeating units (except for the insert in (b)). While the matrix units appear rather uniform in length, the spacer intercepts in such circles show differences. The 2 spacers in a specific eircle may be identical in length ((a), (b) and (c)) but may greatly differ in their absolute lengths among different circles (compare (a) and (c) with (b)). Furthermore, an individual circle may contain 2 spacer intercepts of different lengths (d). The insert in (b) presents a ring that contains 1 matrix unit and an extremely long spacer, which, however, may also include a transcriptionally inactive gene region (total circumference $16 \mu \mathrm{m}$ ). Note also the occasional occurrence of individual lateral fibrils attached to the apparent spacer segments (a). All bars represent $1 \mu \mathrm{m}$.

quantitative analysis of the lengths of the matrix units, i.e. transcriptional units, and the adjacent apparent spacer sections (Fig. 4) showed a rather homogeneous distribution of the matrix unit lengths (Fig. 4 (a)) but widely variable spacer lengths (Fig. 4 (b)), resulting in a corresponding variability of the repeating units (Fig. 4 (c)). The average for the whole population of apparent spacer intercepts in the Dytiscus oocyte rDNA was $4.36 \mu \mathrm{m}$; however, the standard deviation $(2 \cdot 34 \mu \mathrm{m})$ and the range (up to $12 \cdot 2 \mu \mathrm{m}$ ) was much greater than in the matrix units, which may partially 


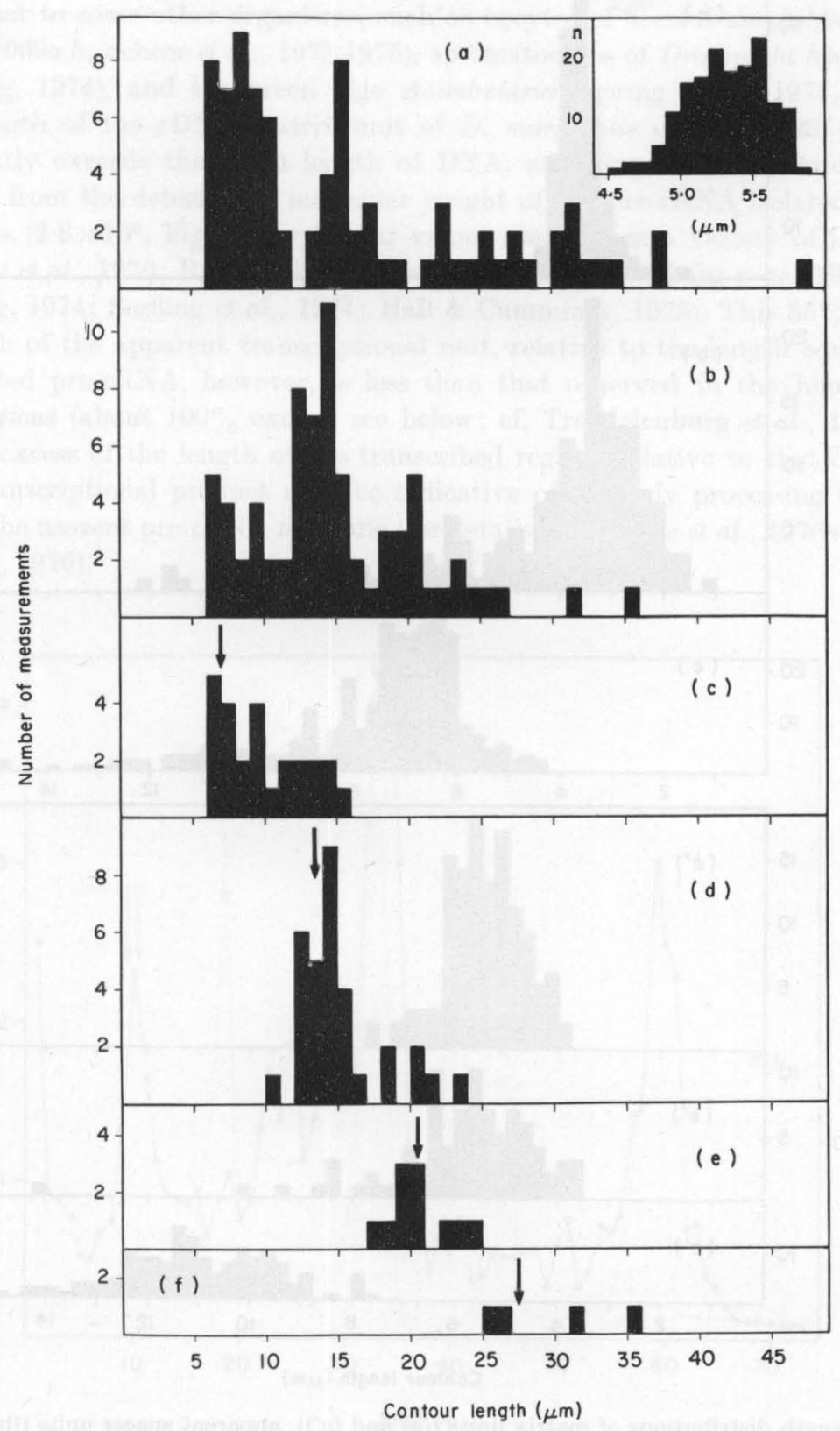

FIG. 3. Comparison of the contour lengths of isolated rDNA circles (a) and transcriptionally active nucleolar chromatin rings (b) from Dytiscus oocytes. Mitochondrial DNA rings found in the same preparation are significantly smaller (mean length $5 \cdot 26 \pm 0 \cdot 34 \mu \mathrm{m}$ ) and have a much narrower length distribution (insert in (a)). The diagram of the contour lengths of circular rDNA containing chromatin (b) includes the circles composed of 1 (c), 2 (d), 3 (e), and 4 (f) repeating units. The arrow in (c) points to the most predominant repeating unit class $(6.95 \mu \mathrm{m})$ as calculated from the data of Fig. 4 (a) to (c), the arrows in (d), (e) and (f) represent multiples thereof. 


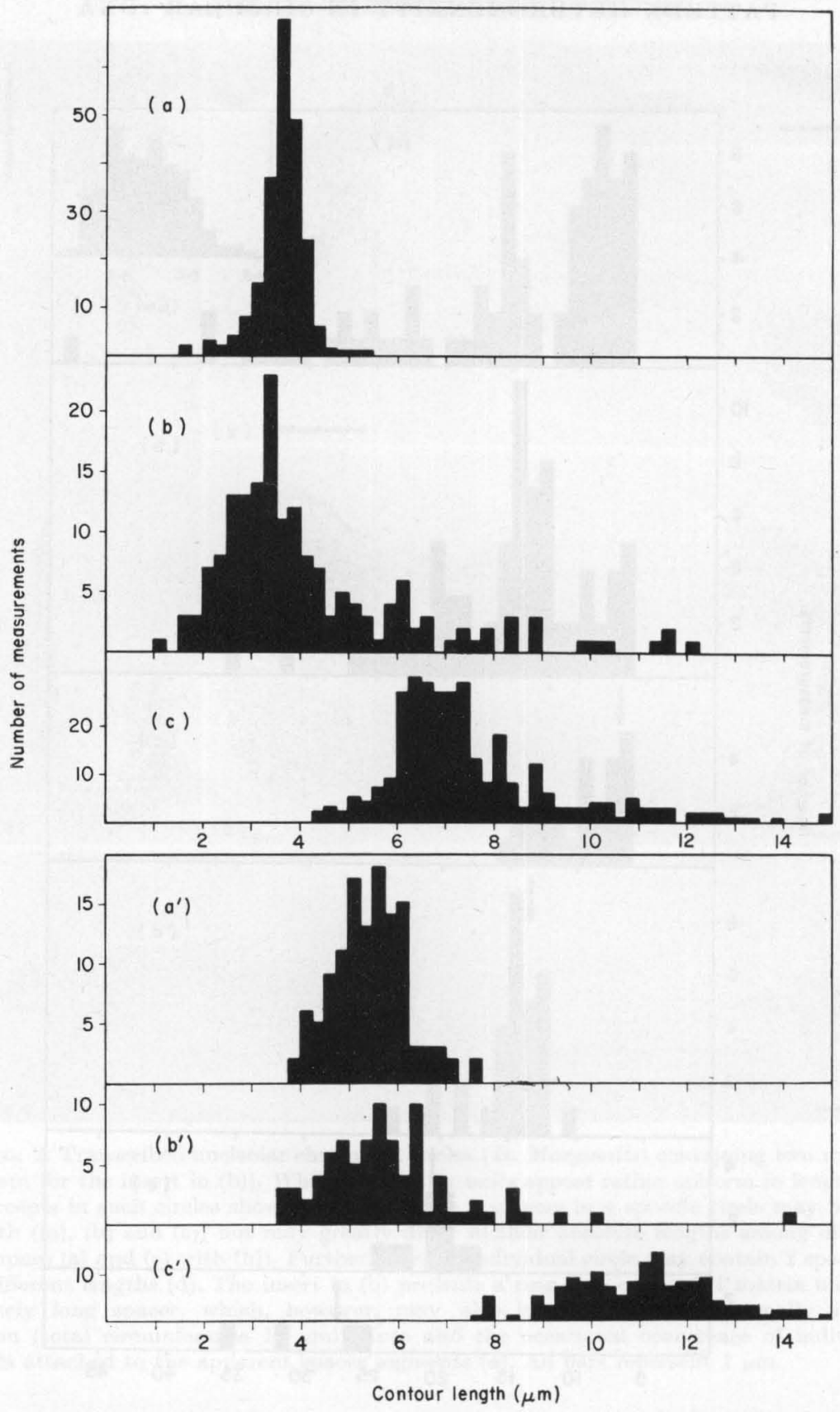

FIG. 4. Length distributions of matrix units ((a) and $\left.\left(a^{\prime}\right)\right)$, apparent spacer units ((b) and (b')), and the resulting repeating units $\left((\mathbf{c})\right.$ and $\left.\left(\mathbf{c}^{\prime}\right)\right)$ as measured in spread transeribed nucleolar chromatin of oocytes from $D$. marginalis ( $(\mathrm{a})$ to $(\mathrm{c}))$ and $A$. domesticus $\left(\left(\mathrm{a}^{\prime}\right)\right.$ to $\left.\left(\mathrm{c}^{\prime}\right)\right)$. In this histogram values from circular as well as from linear strands have been included. In both insect species, the matrix unit lengths show a relatively homogeneous distribution with mean values and standard deviations of $3.57 \pm 0.52 \mu \mathrm{m}$ (a) and $5.41 \pm 0.77 \mu \mathrm{m}\left(\mathrm{a}^{\prime}\right)$. Most $(80 \%)$ of the apparent spacer intercepts of Dytiscus are distributed between 1.5 and $5.5 \mu \mathrm{m}$ with a peak mean of $3.36 \mu \mathrm{m}(\mathrm{b})$, corresponding to a predominant mean peak repeating unit class of $6.95 \mu \mathrm{m}(\mathrm{c}) .20 \%$ out of the measured 175 spacer segments, however, are much longer and very heterogeneous in a broad distribution up to $12 \cdot 2 \mu \mathrm{m}$ (b), resulting in a corresponding heterogeneity of repeating unit lengths (c). An even more pronounced heterogeneity is found in Acheta oocytes $\left(\left(\mathbf{a}^{\prime}\right)\right.$ to $\left.\left(\mathbf{c}^{\prime}\right)\right)$. 
reflect the heterogeneity (Fig. 4 (b)). The most frequent, relatively homogeneous subclass of apparent spacers had a mean (peak) length of $3.36 \mu \mathrm{m}$ (s.D. $0 \cdot 84 \mu \mathrm{m}$ ). In contrast to some other organisms, such as oocytes of several amphibia (Miller \& Beatty, 1969a,b; Scheer et al., 1973,1975), spermatocytes of Drosophila hydei (Meyer \& Hennig, 1974), and the green alga Acetabularia (Spring et al., 1974,1976), the mean length of the rDNA matrix unit of $D$. marginalis oocytes $(3.57 \pm 0.52 \mu \mathrm{m})$ significantly exceeds the mean length of DNA, assuming $B$-conformation, that is expected from the determined molecular weight of the pre-rRNA isolated from the same cells $\left(2.8 \times 10^{6}\right.$, Fig. 5 ; for similar values obtained in a variety of insects see, e.g. Perry et al., 1970; Rubinstein \& Clever, 1971; Trendelenburg et al., 1973; Meyer \& Hennig, 1974; Serfling et al., 1974; Hall \& Cummings, 1975). This 35\% excess of the length of the apparent transcriptional unit, relative to the length equivalent of the isolated pre-rRNA, however, is less than that observed in the house cricket A. domesticus (about 100\% excess, see below; ef. Trendelenburg et al., 1973,1976). Such an excess of the length of the transcribed regions relative to that of the first stable transcriptional product may be indicative of an early processing that takes place in the nascent pre-rRNA molecule (for details see Franke et al., 1976a; Rungger \& Crippa, 1976).

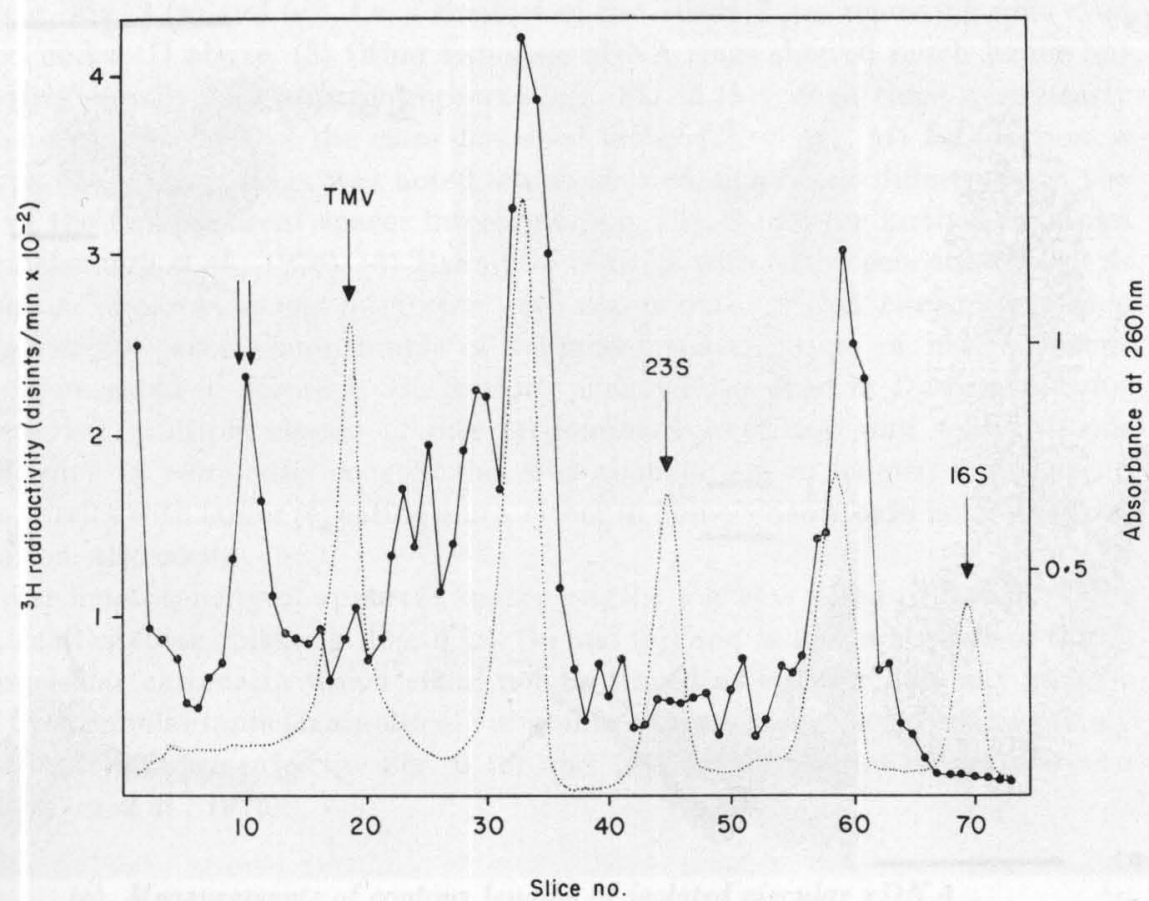

FIG. 5. Gel electrophoretic analysis of $\left[{ }^{3} \mathrm{H}\right]$ uridine-labelled RNA extracted from manually isolated nuclei of Dytiscus oocytes (here from 44 individual nuclei, for further details see Materials and Methods). The pre-rRNA peak (denoted by the double arrow) has an apparent molecular weight of $2.8 \times 10^{6}$ as calibrated against the coelectrophoresed $\left(\ldots \ldots \ldots \ldots, A_{260}\right) E$. coli $23 \mathrm{~S}$ and $16 \mathrm{~S}$ rRNAs (molecular weights $0.525 \times 10^{6}$ and $1.05 \times 16^{6}$ ) and TMV RNA (apparent molecular weight $2.2 \times 10^{6}$; ef. Loening, 1969). Mature $28 \mathrm{~S}$ (molecular weight $1.48 \times 10^{6}$ ) and $18 \mathrm{~S}$ rRNAs $\left(0.71 \times 10^{6}\right)$ are present due to the presence of the nuclear envelope and some contamination of the nuclei by juxtanuclear cytoplasmic material.

- -,${ }^{3} \mathrm{H}$ radioactivity. 
In diplotene oocytes from $A$. domesticus, circular units of transcribed nucleolar chromatin are found less frequently (cf. Trendelenburg et al., 1973), but a variable proportion (from 10 to $30 \%$ ) of the rDNA was routinely recovered in the form of preserved and transcribed rings (Fig. 6). Among such well-identified circles, those containing one or two pre-rRNA genes were predominant (Fig. 6(a),(b) and (c)). This observation, however, does not speak against the occurrence of most of the Acheta rDNA in circles in vivo. A considerable proportion of such circular units might

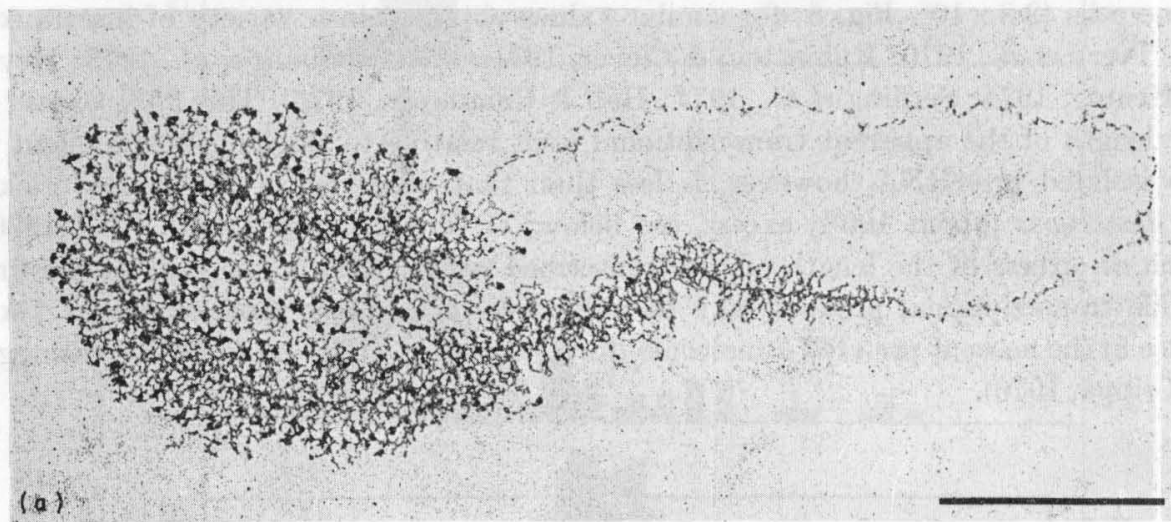

(a)
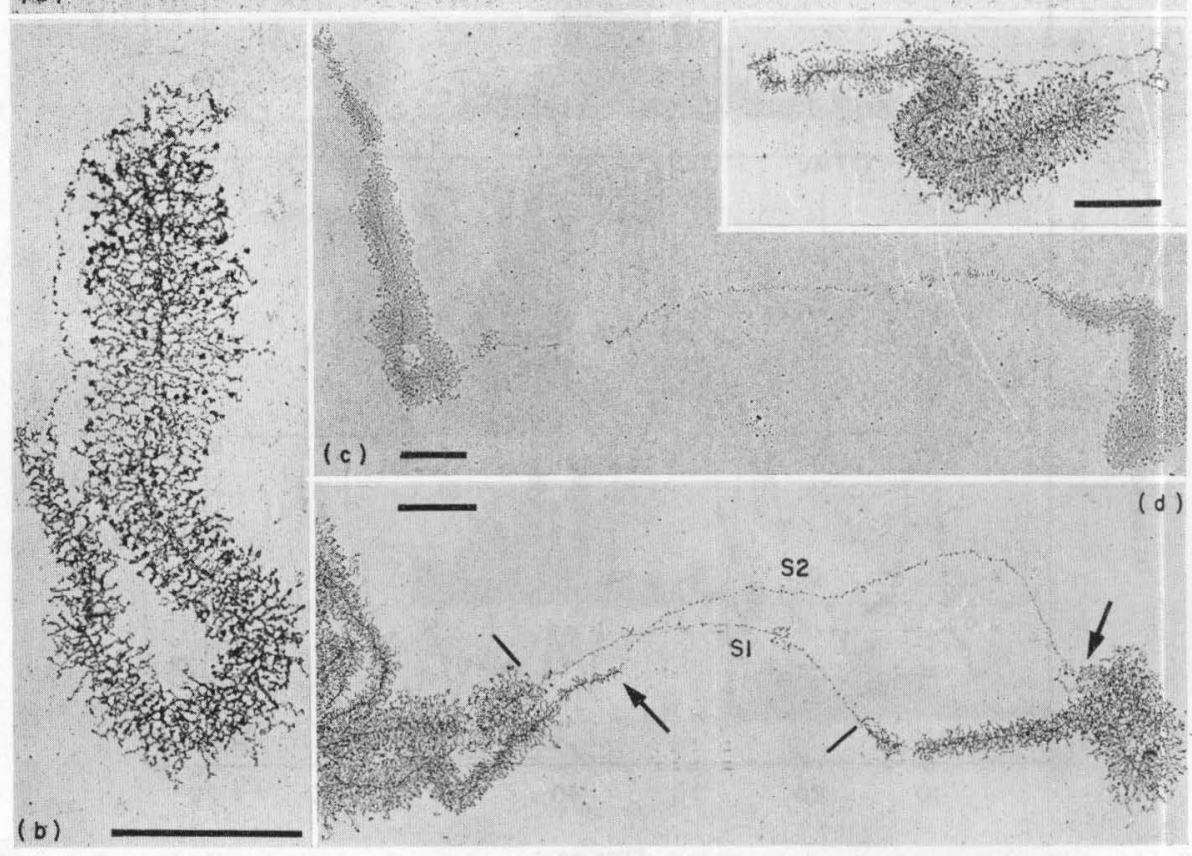

FIG. 6. Spread preparation of nucleolar chromatin from A. domesticus oocyte. Circular units consisting of 1 repeating unit with an approx. 1: 1 length ratio of matrix unit and spacer intercept are shown in (a) and in the insert of (c). (b) A circle with a very short spacer intercept, whereas both the matrix units shown in (c) are separated from each other by a very long spacer segment. The matrix unit in the right part of (d) is included by 2 spacers of greatly different lengths (the shorter one, $\mathrm{S} 1$, is denoted by bars; the longer one, $\mathrm{S} 2$, is demareated by arrows). The free ends of the lateral fibrils in the second half of the matrix units are characterized by densely stained "terminal knobs". The preparation shown in (c) has been metal shadowed after positive staining. Bars indicate $1 \mu \mathrm{m}$. 
have been broken during the preparation, and another moiety of rDNA rings appears to be obscured in such preparations by the high tendency of the exceedingly long matrix units $\left(5 \cdot 41 \pm 0 \cdot 77 \mu \mathrm{m}\right.$; Fig. $\left.4\left(\mathrm{a}^{\prime}\right)\right)$ to aggregate with each other (cf. Trendelenburg et al., 1973,1976). The distribution of the rDNA matrix units was broader in Acheta than in Dytiscus. In addition, heterogeneity of apparent spacer units (mean $5 \cdot 84 \pm 1 \cdot 58 \mu \mathrm{m}$; range from $3 \cdot 5$ to $11 \mu \mathrm{m}$ ) and total repeat units (mean $11 \cdot 13 \pm 1 \cdot 6 \mu \mathrm{m}$; range from $7 \cdot 6$ to $16 \mu \mathrm{m}$ ) was evident (Fig. $4\left(b^{\prime}\right)$ and $\left.\left(c^{\prime}\right)\right)$.

\section{(b) Distribution of lengths of matrix units and apparent spacer intercepts} in individual $r D N A$ circles

When we measured the entire contour lengths of circles of transcribed nucleolar chromatin as well as those of the specific matrix units and apparent spacer intercepts in individual rings from Dytiscus (see e.g. Fig. 3 (b) to (f)), we found a pronounced heterogeneity. Different forms could be distinguished. (1) Different single gene rings could contain apparent spacers of different lengths, ranging from $2 \cdot 3$ to approximately $12 \mu \mathrm{m}$ (e.g. inserts in Figs 1 and $2(\mathrm{~b})$ ), and this resulted in a heterogeneous distribution of the total contour lengths of single gene rings (Fig. 3 (c)). (2) Among the circles consisting of two transcribed pre-rRNA genes, the most prominent type was that containing relatively short apparent spacers both of equal length (e.g. Fig. 2 (a) and (c)), i.e. a doublet of the about $7 \mu \mathrm{m}$ repeating unit class described under (1) above. (3) Other two-gene rDNA rings showed much larger but again nearly equally long apparent spacers (e.g. Fig. 2 (b)); such rings were nearly twice the contour length of the class described under (2), above. (4) In addition, a third type of two-gene rings was noted which showed significant differences in the lengths of the two apparent spacer intercepts (e.g. Fig. 2 (d); for further examples see Trendelenburg et al., 1976). (5) Examples of rings with heterogeneous as well as homogeneous apparent spacer intercepts were also noted in rDNA circles containing three, four or five genes. One example of a "mixed spacer" type of heterogeneous pattern is presented in Figure 1. The various patterns described in Dytiscus oocytes suggested that multiple classes of one predominant repeating unit exist (in our material from $D$. marginalis oocytes this was about $7 \mu \mathrm{m}$ in length) but that, in addition, circles with larger repeating units, either in homogeneous or in heterogeneous combination, also occur.

A similar heterogeneity of apparent spacer lengths was also noted in Acheta rDNA in both small circular units (e.g. Fig. 6 (a), (b) and (c)) and in longer strands of transcribed nucleolar chromatin which could not be traced as circular but may well be derived from circular units (examples of variability of spacer lengths in such seemingly linear strands are presented in Fig. 6 (c) and (d); for further examples see also Trendelenburg et al., 1976).

\section{(c) Measurements of contour lengths of isolated circular $r D N A$}

When we isolated, according to the procedure described by Gall \& Rochaix (1974), DNA from Dytiscus marginalis oocytes that was enriched in rDNA we noted, besides a certain "contamination" of circles of mitochondrial DNA from the juxtanuclear mitochondria (insert in Fig. 3 (a)), that adhered to the isolated nuclei (see Materials and Methods), a wide size range of rDNA circles (Fig. 7). Measurements of the molecular contour lengths (Fig. 3 (a)) revealed a broad heterogeneity. One group of 

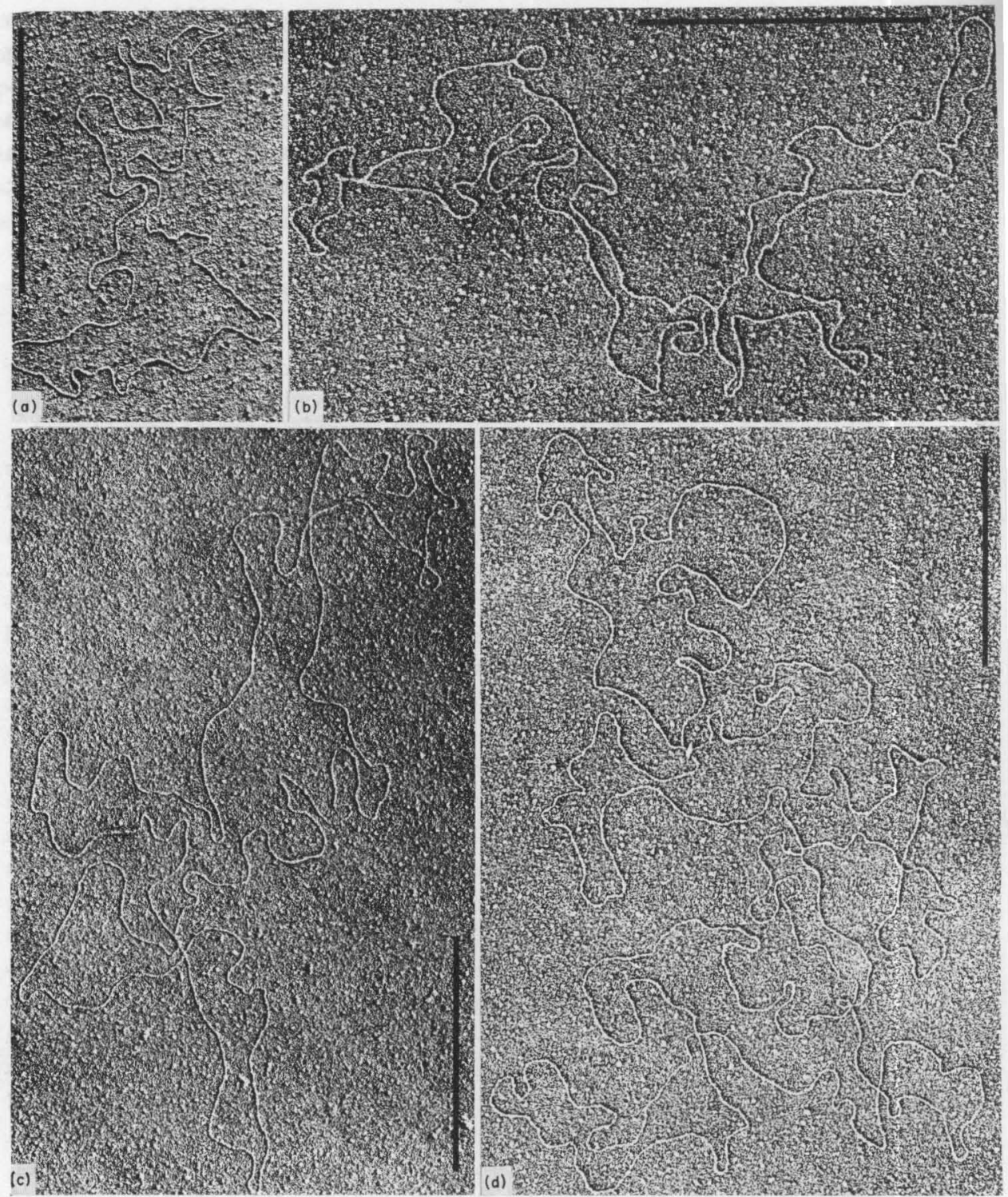

FIG. 7. Electron micrographs of circular rDNA isolated from nuclei of D. marginalis oocytes. Note the differences in molecule length. The contour lengths of the individual eircles shown here are $6.9 \mu \mathrm{m}$ (a), $11.9 \mu \mathrm{m}$ (b), $17.4 \mu \mathrm{m}$ (c), and $31.3 \mu \mathrm{m}$ (d). All bars represent $1 \mu \mathrm{m}$.

small molecules that contained about $25 \%$ of the total circular rDNA was heterogeneous and rather broadly distributed between 6 and $12 \mu \mathrm{m}$. Such relatively small rDNA circles were clearly distinguished from the mitochondrial DNA circles that were characterized by a relatively narrow distribution with a mean length of $5 \cdot 26 \pm$ $0.34 \mu \mathrm{m}$ (Fig. 3(a), insert), corresponding to the sizes of mitochondrial DNA determined in other insects (see e.g. Polan et al., 1973). This class of small rDNA circles appears to correspond to the group of $6 \cdot 3$ to $15 \mu \mathrm{m}$ large single gene rings described in the transcribed nucleolar chromatin (see above). The non-Gaussian mode of 
distribution of the lengths clearly speaks against an homogeneous size class (for a contrasting finding see Gall \& Rochaix, 1974), although the existence of a smallest subclass with a mean length of about $7 \mu \mathrm{m}$ may be significant. Among the larger circles we again noted a broad distribution with only one distinct subclass at a mean molecular length of $15 \cdot 3 \pm 1 \cdot 2 \mu \mathrm{m}$, which might correspond to the two-gene rings with homogeneous short spacers described in the spread transcribed chromatin (mean length about $14 \mu \mathrm{m}$; see above and Fig. $3(\mathrm{~d})$ ). Some of the larger rDNA rings shown in Figs 7 and 3(a) might well correspond to multiples of the most frequent $7 \mu \mathrm{m}$ repeating unit class, as this is also suggested from the measurements of transcribed nucleolar chromatin strands containing three, four (Fig. 3 (e) and (f)) and five genes, but again the broad heterogeneity of molecular sizes was apparent. Seven out of 85 rDNA circles showed conspicuous supercoiling (range of sizes of supercoiled rings from 8.5 to $24 \mu \mathrm{m}$; for supercoiled rDNA in $X$. laevis see Rochaix et al., 1974, and Buongiorno-Nardelli et al., 1976). In addition, five circles with lariat forms were observed.

With rDNA isolated from Acheta oocytes, fewer circles were obtained with our preparative conditions, but a similar, even broader heterogeneity of contour lengths was noted. (range from 7.5 to $16.5 \mu \mathrm{m}$ ).

\section{Discussion}

Electron microscopy of spread preparations of nucleolar chromatin according to the procedure developed by Miller and collaborators (Miller \& Beatty, 1969a,b; Miller \& Bakken, 1972) allows precise and reproducible length measurements of the repeating units of rDNA. Variations in the degree of swelling of the nucleolar material such as those induced by prolonged incubation in the medium and the inclusion of detergents do not result in a significant change of these lengths (cf. Franke et al., $1976 b$ ). When nucleoli from vitellogenic oocytes of $X$. laevis from a variety of shippings and samples, including animals with only one nucleolus organizer, are spread, various size classes of repeating units are found (Scheer, Trendelenburg \& Franke, manuscript in preparation; ef. also Franke et al., 1976b). The two shortest classes of rDNA repeating units observed are 3.45 and $3.70 \mu \mathrm{m}$ (mean peak values), equivalent to $6.9 \times 10^{6}$ and $7 \cdot 4 \times 10^{6}$ molecular weight of rDNA, values which correspond closely to those calculated from the sizes of fragments obtained after cleavage of rDNA with restriction endonuclease EcoRl (Morrow et al., 1974; Wellauer et al., 1974,1976). This indicates that lengths of rDNA intercepts as revealed in spread chromatin preparations correlate with measurements in isolated rDNA using electron microscopy and agarose gel electrophoresis. The validity of the analysis of rDNA patterns in electron micrographs of spread preparations of transcribed nucleolar chromatin is further supported by the demonstration of strands with different patterns (genespacer lengths) on the same grid. For example, rDNA strands with only one size class of short spacers can be distinguished from other strands with a different, yet homogeneous class of longer spacers as well as from strands characterized by a broad distribution of spacer lengths, i.e. with marked intrafilar heterogeneity (cf. Spring et al., 1976). When we mixed, in control experiments, isolated nuclear contents from diverse origins such as from Acheta oocytes and from vegetative Acetabularia cells on a grid, we could easily identify the species-specific rDNA patterns. Therefore, artificial variation in the degree of stretching or shortening of matrix units, spacer units and 
total repeating units must be negligible. Comparisons of transcribed and untranscribed adjacent gene regions in only partially active nucleoli (cf. Scheer et al., 1976) have further shown that the length of the rDNA repeating unit as revealed in spread nucleolar chromatin does not significantly change during transcription (Franke et $a l ., 1976 b)$.

The present study shows, in the oocytes of two taxonomically quite distant insect species, that the pattern of transcribed pre-rRNA genes and (apparently untranscribed) interspersed spacer regions in amplified circular rDNA is not constant but displays a significant intraspecies heterogeneity. Our finding that this pattern can greatly differ in different rDNA circles seems to be in conflict with the report by Gall \& Rochaix (1974). From their measurements of contour lengths of circles of isolated rDNA from two water beetle species these authors concluded that all amplified rDNA circles represent multiples of one and only one repeating unit, which they found to have a molecular weight of $19.2 \times 10^{6}$ in their material of $D$. marginalis and about $10^{7}$ in Colymbetes fuscus (molecular weights of double strand DNA). Although their measurements already exhibited some heterogeneity of molecular sizes that would not fit in this multiplicity scheme, the variance of their data and ours is obvious: neither did we find a uniform repeating unit, nor does the most frequent size class of repeating units observed in our D. marginalis material, i.e. the approximately $14 \times 10^{6}$ molecular weight (see above), correspond to the repeating unit described by these authors. The reasons for this diversion are unknown. (A similar discrepancy exists with respect to the existence of a uniform repeating unit in rDNA from $X$. laevis; for references see below.) However, it may well be that the rDNA "gene-spacer" ratios and patterns, especially with respect to the relative proportions of subclasses of different rDNA patterns present in a specific sample after a specific preparation, reflect true variations among different races and regional varieties, which perhaps result from quantitative differences in the contributions of the various nucleolus organizer regions during the amplification process (see below). Similar differences have recently been noted among different samples and different nuclei in some green algae of the Dasycladaceae (for a detailed discussion see Spring et al., 1976) and among different shipments of $X$. laevis (Wellauer et al., 1976).

From our measurements of the transcriptional units in the spread nucleolar chromatin, it is further evident that in both species examined the apparent spacer intercepts are much more heterogeneous in size than the transcribed and fibrilcovered pre-rRNA gene regions (matrix units; see also Trendelenburg, 1974). This finding concurs with the recent demonstrations of length heterogeneity of the apparent spacer intercepts (i.e. in this case the "non-transcribed spacer", sensu Brown et al., 1972 ) in both chromosomal and amplified rDNA copies of two species of the genus Xenopus (Morrow et al., 1974; Wellauer \& Dawid, 1974; Wellauer et al., 1974,1976; Wellauer \& Reeder, 1975; see also Scheer et al., 1973, and Franke et al., 1976b) but is seemingly at variance with other reports which claim that the amplified rDNA circles of $X$. laevis contain multiples of one basic repeating unit $\left(8 \times 10^{6}\right.$ molecular weight according to Hourcade et al., 1973 and Buongiorno-Nardelli et al., 1976; 7.5 $\times 10^{6}$ according to Rochaix \& Bird, 1975; $7 \cdot 25 \times 10^{6}$ according to Rochaix et al., 1974 and Bakken, 1975; note, however, the indication of some heterogeneity in the actual measurements published by some of these authors).

As to the general problem of the significance and origin of the intraspecies size heterogeneities in rDNA pattern, we would like to distinguish between the following 
qualitatively different situations. (1) Random heterogeneity in all rDNA strands, which allows for a broad range of values without preferential subclasses; (2) random heterogeneity in some rDNA strands and homogeneity or heterogeneity with distinct subclasses in other strands (see below); (3) heterogeneity in which distinct subclasses of rDNA intercepts are located on different rDNA strands such that the pattern of individual rDNA strands is homogeneous (inter-axial heterogeneity); (4) heterogeneity with different classes of rDNA units that occur on the same strand (intraaxial or intrafilar heterogeneity). The present study as well as the findings in the green alga Acetabularia and related genera (Spring et al., 1974, 1976; Berger \& Schweiger, $1975 a, b)$ provide examples of the occurrence of rDNA pattern heterogeneity of the latter forms ((3) and (4)). The analyses of rDNA patterns in X. laevis and X. muelleri with restriction endonucleases (Morrow et al., 1974; Wellauer et al., 1974; Wellauer \& Reeder, 1975) have demonstrated fragments of distinct size classes, which also speaks for these two forms of heterogeneity in subclasses. Wellauer et al. (1976) have described that in the same animal (X. laevis) amplified rDNA molecules are much more homogeneous in spacer lengths than chromosomal rDNA strands, which emphasizes the dominance of heterogeneity of type (3) in the amplified rDNA. However, our data in insect as well as in amphibian (cf. Scheer et al., 1973) oocytes and in some green algae (for references see above) do not rule out but rather tend to support the existence of some random heterogeneity of the forms mentioned under (1) and (2), at least in some specific nucleolar chromatin strands (Spring et al., 1976). As a consequence of this terminological differentiation, heterogeneity and/or homogeneity of rDNA patterns can be adequately discussed and defined only in the individual rDNA unit, be it linear or circular, since analyses of total rDNA would not distinguish between the various forms of inter- and intra-axial heterogeneity described. The functional meaning of the occurrence of various rDNA patterns within one species and even within one nucleolar chromatin strand remains unclear, in that the general function of the "spacer" regions as such is not yet understood. The fact that such interspersed intercepts between pre-rRNA genes are, in most cases, not simultaneously transcribed into large RNA molecules (molecular weight greater than about $15 \times 10^{4}$ ) does not mean that they do not contain genetic information. Some recent observations of occasional transcriptional events in apparent spacer intercepts between pre-rRNA matrix units (Figs 2 and 6 ; and see, e.g. Scheer et al., 1973; Trendelenburg et al., 1973; Spring et al., 1974; Franke et al., 1976a; Rungger \& Crippa, 1976) indeed suggest that parts of the "non-transcribed spacers" of rDNA (sensu Brown et al., 1972) can be transcribed, irrespective of whether this transcription is related to that of the adjacent pre-rRNA genes (for detailed discussions seereferences quoted above). Moreover, the observations of occasional and irregularly arranged 120 to $180 \AA$ large granules that are attached to such apparent spacer regions and are structurally and eytochemically not distinguished from the RNA polymerase molecules within the matrix units (Miller \& Beatty, 1969a; Scheer et al., 1976; Franke et al., 1976b) might suggest that such spacer intercepts provide at least sites for a specific attachment of RNA polymerase A, perhaps in a transcriptionally inactive state.

One inevitable conclusion from the demonstrations of a variety of heterogeneous forms of gene-spacer length patterns in amplified rDNA, even in small rDNA circles, is that such nucleolar components must have been derived from different chromosomal regions. In view of the low numbers of nucleolar organizers involved in the 
rDNA amplification in various amphibia including Xenopus (for references see MacGregor, 1973) and in A. domesticus (Lima-de-Faria et al., 1973; as to the less clear situation in Dytiscus see references in Gall et al., 1969), we think it more likely that the observed heterogeneities of amplified rDNA at least partly reflect the heterogeneities of different regions within one nucleolus organizer. This notion that amplified rDNA molecules, especially the small circles, can be derived from different regions of the same organizer is also supported by measurements in spread nucleolar chromatin (Scheer, Trendelenburg \& Franke, manuscript in preparation) and by demonstrations of spacer length heterogeneity after cleavage of rDNA with restriction endonucleases (see Wellauer et al., 1974) in the $+/ 0$ nucleolus organizer mutant of $X$. laevis.

The rDNA of oocytes of Acheta and Dytiscus seems to differ from the rDNA of amphibian oocytes and the primary nuclei of the green alga Acetabularia in that the insect amplified rDNA shows a larger proportion of molecules with tandem heterogeneity of spacer lengths. In spread preparations of transcriptionally active nucleolar chromatin from amphibian oocytes, one finds a striking predominance of strands with identical repeating units, i.e. intra-axial homogeneity (see, e.g. Miller \& Beatty, $1969 a, b$; Scheer et al., 1973, 1976). This concurs with the analysis by Wellauer et al. (1976) of oocytes from $X$. laevis using rDNA fragments obtained with restriction endonuclease as well as heteroduplex molecules containing cloned rDNA fragments including spacer regions. These authors found that in amplified rDNA only $13 \%$ of neighbouring repeating units are non-identical and conclude "that most if not all repeats on the same strand of amplified rDNA (neighbor repeats) have lengths which are not distinguishable". Similarly, we have described in Acetabularia the predominance of rDNA strands in which adjacent repeating units are similar if not identical in length but that also a small proportion of strands exists that show marked tandem heterogeneity of repeating units (Spring et al., 1976). The reason for the existence of rDNA circles with heterogeneous lengths of apparent spacer intercepts in insects is not clear. Assuming that amplification in insect oocytes also takes place via a "rolling circle" mechanism, as has been reported for amphibia (Hourcade et al., 1973; Rochaix et al., 1974; Bakken, 1975), this heterogeneity in length would require the existence of some replicating circles that contain several non-identical repeating units.

The correspondence observed between the contour lengths of the isolated rDNA circles and those of the transcribed nucleolar chromatin rings, in particular those of the smallest size classes, also argues against the packing of much, if any, of this DNA into nucleosomes during transcription (for a detailed discussion see Franke et al., 1976b). In support of this conclusion is the obvious absence of nucleosomal structures in the transcribed nucleolar chromatin strands as revealed by the spreading technique, which allows an excellent demonstration of authentic nucleosomes (for references see Olins \& Olins, 1974; McKnight \& Miller, 1976; Franke et al., 1976b).

We thank G. Krohne, Dipl. Biol. (this institute) for his co-operation in the analysis of the pre-rRNA and Miss Marianne Winter for skilful technical assistance. The work has been partially supported by the Deutsche Forschungsgemeinschaft (Bonn-Bad Godesberg, grant Sche 157/2). Parts of this work have been presented at the Fourth Nucleolar Workshop, September 15-20, 1975, held at Varna, Bulgaria. 


\section{REFERENCES}

Angelier, N. \& Lacroix, J. C. (1975). Chromosoma, 51, 323-335.

Bakken, A. H. (1975). J. Histochem. Cytochem. 23, 463-474.

Berger, S. \& Schweiger, H. G. (1975a). Mol. Gen. Genet. 139, 269-275.

Berger, S. \& Schweiger, H. G. (1975b). Planta, 127, 49-62.

Birnstiel, M. L., Chipchase, M. \& Speirs, J. (1971). Progr. Nucl. Acid Res. 11, 351-389.

Bohnert, H. J., Schiller, B., Böhme, R. \& Sauer, H. W. (1975). Eur. J. Biochem. 57, 361369.

Brown, D. D. \& Sugimoto, K. (1974). Cold Spring Harbor Symp. Quant. Biol. 38, 501505.

Brown, D. D., Wensink, P. C. \& Jordan, E. (1972). J. Mol. Biol. 63, 57-73.

Buongiorno-Nardelli, M., Amaldi, F. \& Lava-Sanchez, P. A. (1976). Exp. Cell Res. 98, 95-103.

Cave, M. D. (1972). J. Cell Biol. 55, 310-321.

Cave, M. D. (1973). Chromosoma, 42, 1-22.

Davis, R. W., Simon, M. \& Davidson, R. (1971). Methods Enzymol. 21, 413-428.

Dawid, I. B., Brown, D. D. \& Reeder, R. H. (1970). J. Mol. Biol. 51, 341-360.

Engberg, J., Christiansen, G. \& Leick, V. (1974). Biochem. Biophys. Res. Commun. 59, 1356-1365.

Espejo, R. T., Canelo, E. S. \& Sinsheimer, R. L. (1969). Proc. Nat. Acad. Sci., U.S.A. 63, 1164-1168.

Forsheit, A. B., Davidson, N. \& Brown, D. D. (1974). J. Mol. Biol. 90, 301-314.

Franke, W. W., Scheer, U., Spring, H., Trendelenburg, M. F. \& Krohne, G. (1976a). Exp. Cell Res. 100, 233-244.

Franke, W. W., Scheer, U., Trendelenburg, M. F., Spring, H. \& Zentgraf, H. (1976b). Cytobiologie 13, 401-434.

Gall, J. G. (1974). Proc. Nat. Acad. Sci., U.S.A. 71, 3078-3081.

Gall, J. G. \& Rochaix, J. D. (1974). Proc. Nat. Acad. Sci., U.S.A. 71, 1819-1823.

Gall, J. G., Macgregor, H. C. \& Kidston, M. E. (1969). Chromosoma, 26, 169-187.

Hall, T. J. \& Cummings, M. R. (1975). Develop. Biol. 46, 233-242.

Hourcade, D., Dressler, D. \& Wolfson, J. (1973). Proc. Nat. Acad. Sci., U.S.A. 70, 29262930.

Lang, D. \& Mitani, M. (1970). Biopolymers, 9, 373-379.

Lima-de-Faria, A., Daskaloff, S. \& Enell, A. (1973). Hereditas, 73, 99-118.

Loening, U. E. (1969). Biochem. J. 113, 131-138.

Macgregor, H. C. (1973). Nature New Biol. 246, 81-82.

McKnight, S. L. \& Miller, O. L. (1976). Cell, 8, 305-319.

Meyer, G. F. \& Hennig, W. (1974). Chromosoma, 46, 121-144.

Miller, O. L. \& Bakken, A. H. (1972). Acta Endocrinol. 168 (suppl.), 155-177.

Miller, O. L. \& Beatty, B. R. (1969a). J. Cell. Physiol. 74 (suppl. I), 225-232.

Miller, O. L. \& Beatty, B. R. (1969b). Genetics, 61 (suppl.), 134-143.

Morrow, J. F., Cohen, S. N., Chang, A. C. Y., Boyer, H. W., Goodman, H. M. \& Helling, R. B. (1974). Proc. Nat. Acad. Sci., U.S.A. 71, 1743-1747.

Olins, A. L. \& Olins, D. E. (1974). Science, 183, 330-332.

Perry, R. P., Cheng, T. Y., Freed, J. J., Greenberg, J. R., Kelley, D. E. \& Tartof, K. D. (1970). Proc. Nat. Acad. Sci., U.S.A. 65, 609-616.

Polan, M. L., Friedman, S., Gall, J. G. \& Gehring, W. (1973). J. Cell Biol. 56, 580-589.

Rochaix, J. D. \& Bird, A. P. (1975). Chromosoma, 52, 317-327.

Rochaix, J. D., Bird, A. P. \& Bakken, A. H. (1974). J. Mol. Biol. 87, 473-487.

Rubinstein, L. \& Clever, U. (1971). Biochim. Biophys. Acta, 246, 517-529.

Rungger, D. \& Crippa, M., (1976). Progr. Biophys. Mol. Biol. in the press.

Scheer, U., Trendelenburg, M. F. \& Franke, W. W. (1973). Exp. Cell Res. 80, 175-190.

Scheer, U., Trendelenburg, M. F. \& Franke, W. W. (1975). J. Cell Biol. 65, 163-179.

Scheer, U., Trendelenburg, M. F. \& Franke, W. W. (1976). J. Cell Biol. 69, 465-489.

Serfling, E., Maximowsky, L. F. \& Wobus, U. (1974). Eur. J. Biochem. 45, 277-289.

Spring, H., Trendelenburg, M. F., Scheer, U., Franke, W. W. \& Herth, W. (1974). Cytobiologie, 10, 1-65. 
Spring, H., Krohne, G., Franke, W. W., Scheer, U. \& Trendelenburg, M. F. (1976). J. Microscop. Biol. Cell, 25, 107-116.

Trendelenburg, M. F. (1974). Chromosoma, 48, 119-135.

Trendelenburg, M. F., Scheer, U. \& Franke, W. W. (1973). Nature New Biol. 245, 167-170.

Trendelenburg, M. F., Spring, H., Scheer, U. \& Franke, W. W. (1974). Proc. Nat. Acad. Sci., U.S.A. 71, 3626-3630.

Trendelenburg, M. F., Franke, W. W., Spring, H. \& Scheer, U. (1975). In Proc. 9th F'EBS Meeting (Hidvégi, E. J., Sümegi, J. \& Venetianer, P., eds), vol. 33, pp. 159-168, Akademiai Kiado, Budapest \& North-Holland Publishing Co., Amsterdam.

Trendelenburg, M. F., Franke, W. W. \& Scheer, U. (1976). Differentiation, in the press.

Ullman, J. S., Lima-de-Faria, A., Jaworska, H. \& Bryngelsson, T. (1973). Hereditas, 74, 13-24.

Wellauer, P. K. \& Dawid, I. B. (1974). J. Mol. Biol. 89, 379-395.

Wellauer, P. K. \& Reeder, R. H. (1975). J. Mol. Biol. 94, 151-161.

Wellauer, P. K., Reeder, R. H., Caroll, D., Brown, D. D., Deutch, A., Higashinakagawa, T. \& Dawid, I. B. (1974). Proc. Nat. Acad. Sci., U.S.A. 71, 2823-2827.

Wellauer, P. K., Reeder, R. H., Dawid, I. B. \& Brown, D. D. (1976). J. Mol. Biol. 105, 487-506.

Wensink, P. C. \& Brown, D. D. (1971). J. Mol. Biol. 60, 235-247. 